to $2.13 \%$ with mean of $0.67 \pm 0.59 \%$. A high significant difference was found between SLE pts and controls $p=0.001$, an insignificant rise was seen in SLE pts compared to T1DM pts $p=0.157$. A high significant increase in Th9 was found in severe SLE: mean of $3.74 \pm 1.15 \%$ than in pts with mild to moderate SLE: mean $0.94 \pm 0.88 \% \mathrm{p}=<0.001$

IL9 level was highly increased in SLE pts: mean of $42.83 \pm 23.98 \mathrm{pg} / \mathrm{ml}$ than both control gps. In healthy controls the mean was $8.54 \pm 13.27$, while in T1DM with mean of $29.17 \pm 16.09 \mathrm{pg} / \mathrm{ml}$. A high significant difference was found between SLE pts and normal controls $p<0.001$ but an insignificant rise with T1DM $p=0.294$. A high significant increase in IL9 in pts with severe ds compared to mild to moderate pts $p<0.001$. A significant direct correlation between Th9 \& IL9 and SLEDAI/105 A significant direct correlation between damage index and Th9 $\mathrm{p}=0.040$ but not IL9 $\mathrm{p}=0.053$

In SLE no significant relation between Th9 or IL9 \& clinical manifestations or disease duration. A direct correlations between Th9 \& ESR $p=0.046$ and CRP $\mathrm{p}=0.025$, a significant correlation between IL 9 and CRP $p=0.033$, no correlations between Th9\&IL9 level and anti-dsDNA $p=0.593 \& 0.4$ Significant direct correlation between Th9 and IL9 in T1DM pts, still no correlation with glycemic profile. IL9 levels were significantly increased in SLE with elevated CRP $p=0.033$ \& the $\%$ of Th9 cells were increased with elevated ESR and CRP $\mathrm{p}=0.025,0.046$

Conclusion: In SLE pts; IL9 level and Th9 cells expression were significantly elevated compared to healthy controls. IL9 levels and the percentages of Th9 directly correlated with the SLE disease activity. IL9 levels also were significantly increased in T1DM pts compared to controls,but they were less expressed than in SLE. This suggests an important role of IL9 in the pathogenesis AIDs as SLE

REFERENCES:

[1] Tahernia L et al. Cytokines in SLE: their role in pathogenesis of disease and possible therapeutic opportunities. Rheum Res 2017

Disclosure of Interests: None declared

DOI: 10.1136/annrheumdis-2021-eular.3946

\section{AB0346 $\quad$ RHUPUS SYNDROME: CLINICAL ANALYSIS OF 21 PATIENTS}

M. Ben Brahim ${ }^{1}$, S. Daada ${ }^{1}$, M. Elghali ${ }^{2}$, J. Mahbouba $^{3}$, N. Bergaoui ${ }^{3}$, S. Hammami ${ }^{1}{ }^{1}$ Fattouma Bourguiba University Hospital, Internal Medicine, Monastir, Tunisia; ${ }^{2}$ Fattouma Bourguiba University Hospital, Immunology, Monastir, Tunisia; ${ }^{3}$ Fattouma Bourguiba University Hospital, Rhumatology, Monastir, Tunisia

Background: Rhupus syndrome is a clinical condition characterized by a combination of the clinical and immunologic features of rheumatoid arthritis (RA) and systemic lupus erythematosus (SLE) (1). However, this definition did not establish whether Rhupus syndrome is a distinct clinical entity or is an overlap between RA and SLE. To date, fewer than 200 cases of Rhupus have been reported (1)

Objectives: Our purpose was to analyze the clinical and serological characteristics of patients with Rhupus and compare them with patients with SLE.

Methods: We undertook a retrospective study covering 10 years (2009 to 2019) of experience in our University Hospital: Department of Internal Medicine and department of Rheumatology. 238 patients hospitalized were evaluated during this period. The clinical and laboratory parameters of 21 patients with Rhupus syndrome were compared with those of 217 SLE without RA patients

Results: Twenty one patients were included (all fulfilled ACR criteria for SLE as well as for RA). They were 19 female and 2 male. The mean age of patients at the disease onset was 43.5 years. Among these, 9 patients $(42.8 \%)$ were diagnosed with RA at the onset of disease and then developed SLE over an average period of 6.2 years. Six patients $(28.5 \%)$ had a diagnosis of SLE prior to the RA diagnosis with an interval of 8.6 years between diagnoses. Six patients $(28.5 \%)$ were diagnosed with RA and SLE concomitantly. Patients with Rhupus experienced more often arthritis, joint swelling, morning stiffness and joint erosions then patients with SLE alone $(p<0.001)$. Comparison of extra-articular manifestations showed that, renal involvement was less often in patients with Rhupus when compared to patients with SLE-alone. There were no significant differences in the prevalence of malar rash, serositis, neurological and hematological features between the Rhupus and SLE-alone groups. Rheumatoid factor and anticyclic citrullinated peptide (CCP) antibody were significantly more prevalent in the Rhupus group than in the control group. The incidences of increased erythrocyte sediment rate (ESR) and C-reactive protein (CRP) were also significantly higher in Rhupus patients. (Table 1)
Table 1. Comparison of clinical and biological features between Rhupus Syndrome (Rhupus Group) and SLE-alone patients (Control Group).

\begin{tabular}{lccc} 
& Rhupus Group & Control Group & \\
& $\mathrm{N}=21(\%)$ & $\mathrm{N}=217(\%)$ & $\mathrm{p}$ \\
\hline Malar rash & $12(57.1)$ & $120(55.3)$ & 0.87 \\
serositis & $6(28.5)$ & $47(21.7)$ & 0.46 \\
Lupus nephritis & $4(19)$ & $56(25.8)$ & 0.49 \\
Hemolytic anemia & $1(4.7)$ & $8(3.6)$ & 1 \\
Leucopenia & $8(38)$ & $90(41.4)$ & 0.15 \\
Thrombopenia & $4(19)$ & $27(12.4)$ & 1 \\
Polyarthritis & $21(100)$ & $54(24.8)$ & $<0.001$ \\
Erosion of joint & $21(100)$ & 0 & $<0.001$ \\
Increased ESR & $20(95.2)$ & $155(71.4)$ & 0.019 \\
Increased CRP & $18(85.7)$ & $134(40.5)$ & $<0.001$ \\
Anti-DNA antibodies & $16(76.1)$ & $63(29)$ & 0.61 \\
Anti-Sm antibodies & $6(28.5)$ & $52(23.9)$ & 0.57 \\
Rhumatoide Factor & $12(57.1)$ & $14(6.5)$ & 0.04 \\
Anti-CCP antibody & $6(28.5)$ & & 0.027 \\
\hline
\end{tabular}

CRP: C-reactive protein, ESR: erythrocyte sediment rate, Anti-CCP: anticyclic citrullinated peptide antibody.

Conclusion: Patients sharing features of both RA and SLE have been observed infrequently. In fact, the incidence of Rhupus syndrome in SLE patients is estimated at $1.3 \%(2)$. In our study, this incidence was $8.8 \%$. The findings of our study are consistent with those of most studies with respect to the frequencies arthritis, lupus related features, and serological disorders $(2,3)$.

\section{REFERENCES:}

[1] Rubini E, Foddai SG, Radin M, Cecchi I, Rossi D, Sciascia S, et al. Ab1177 How to Define Rhupus Syndrome: Systematic Review of the Current Literature. Ann Rheum Dis. 2019 Jun 1;78(Suppl 2):2049-50.

[2] Li J, Wu H, Huang X, Xu D, Zheng W, Zhao Y, et al. Clinical Analysis of 56 Patients with Rhupus Syndrome: Manifestations and Comparisons with Systemic Lupus Erythematosus. Medicine(Baltimore). 2014; 93(10): 958-63.

[3] Liu T, Li G, Mu R, Ye H, Li W, Li Z. Clinical and laboratory profiles of rhupus syndrome in a Chinese population: a single-centre study of 51 patients. Lupus. 2014;23(9):958-63.

Disclosure of Interests: None declared

DOI: 10.1136/annrheumdis-2021-eular.3969

\section{AB0347 RENAL BIOPSY IN PATIENTS WITH SYSTEMIC LUPUS ERYTHEMATOSUS: IS IT ONLY LUPUS NEPHRITIS?}

E. Duran ${ }^{1}$, T. Yıldırım², U. Kalyoncu ${ }^{1}$, A. Taghiyeva ${ }^{3}$, E. Bilgin ${ }^{1}$, E. Arzu Sağlam ${ }^{4}$, M. Üner ${ }^{4}$, J. Jabrayilov ${ }^{2}$, E. C. Bolek ${ }^{1}$, C. Önal ${ }^{2}$, B. Farisoğulları ${ }^{1}$, N. S. Koç ${ }^{2}$, G. K. Yardımcı ${ }^{1}$, S. Girgin ${ }^{2}$, G. Ayan ${ }^{1}$, Z. Özsoy ${ }^{1}$, G. Sandal Uzun ${ }^{1}$, L. Kılıçç, S. R. Yılmaz², A. Akdoğan ${ }^{1}$, S. A. Bilgen ${ }^{1}$, O. Karadag ${ }^{1}$, S. Kiraz ${ }^{1}$, B. Altun ${ }^{2}$, Y. Erdem ${ }^{2}$, M. ArıcI ${ }^{2}$ A. İ. Ertenli' ${ }^{1}{ }^{1}$ Hacettepe University School of Medicine, Department of Internal Medicine, Division of Rheumatology, Ankara, Turkey; ${ }^{2}$ Hacettepe University School of Medicine, Department of Internal Medicine, Division of Nephrology, Ankara, Turkey; ${ }^{3}$ Hacettepe University School of Medicine, Department of Internal Medicine, Ankara, Turkey; ${ }^{4}$ Hacettepe University School of Medicine, Department of Department of Pathology, Ankara Turkey

Background: Renal biopsy is a cornerstone in the diagnosis and management of renal involvement in patients with systemic lupus erythematosus (SLE). However, non-lupus nephritis has been also observed in SLE patients with renal disease (1).

Objectives: The aim of this study was to draw attention to the causes of non-lupus nephritis in SLE patients with kidney biopsy.

Methods: This retrospective, descriptive study included 139 SLE patients who had at least one kidney biopsy between 2001 and 2020. All patients had fulfilled the SLICC or EULAR/ACR criteria for SLE. According to the pathology report results, 116 of the patients were diagnosed with lupus nephritis (LN), 18 patients had non-lupus nephritis, 2 biopsies were normal, and 3 biopsies were insufficient. Demographics, SLE disease duration, and renal biopsy diagnosis were derived from our hospital medical records.

Results: Of the 23 patients (female:18/male:5), the mean age at the SLE diag nosis was 30.5 years and the median SLE disease duration was 8.5 (11.6) years Pathologic report findings were compatible with focal segmental glomerulosclerosis in 6 patients, membranous nephropathy with no cellular proliferation and inflammation in 4 patients, thrombotic microangiopathy in 3 patients, IgM nephropathy in 2 patients, tubulointerstitial nephritis in 2 patients, and proliferative glomerulonephritis with monoclonal lgG deposits in one patient. There were no different for SLE manifestation in both gropus. LN vs other renal pathologies 
laboratory comparing as follow: ANA (+) $\geq 1: 32089(76.7 \%)$ vs $14(60.9 \%)$, APS antibodies $31(33.7 \%)$ vs 8 (57.1), anti-Sm (+) 8 (11.8\%) vs $1(4.3 \%)$ were similar for LN and other renal pathologies, but anti-ds-DNA positivity $94(84.7 \%)$ vs 10 (50\%), median ds-DNA level 421 (591) vs 150 (340) and low level of C3 and C4 were more frequent in $L N(p<0.001 ; p=0.005 ; p<0.001$, respectively). In addition, the rate of active urinary sediment and renal SLEDAI score were significantly high in LN patients.

Conclusion: Various renal lesions unrelated to lupus nephritis can be observed in SLE patients. Renal biopsy plays a critical role in identifying these lesions, which may have prognostic and therapeutic implications distinctive from those of lupus nephritis. Also, anti ds-DNA positivity/level, low C3 and C4, active urinary sediment and renal SLEDAI scores may give us some clues in terms of renal pathology for SLE patients. Moreover, almost half of the patients without LN in renal biopsy have anti ds-DNA positivity.

REFERENCES:

[1] Howell DN. Renal biopsy in patients with systemic lupus erythematosus: Not just lupus glomerulonephritis! Ultrastruct Pathol. 2017 Mar-Apr;41(2):135-146.

Table 1. Demographic, clinical characteristics and results of patients with and without lupus nephritis

\begin{tabular}{lccc}
\hline Variables & Lupus nephritis & Other pathologies & \\
& & & \\
& $(\mathbf{N}=116)$ & $(\mathbf{N}=23)$ & $\mathbf{P}$ \\
\hline Age at the SLE diagnosis, years & $22.5 \pm 13.1$ & $30.5 \pm 14.5$ & $\mathbf{0 . 0 0 6}$ \\
Sex, female & $93(80.2)$ & $18(78.3)$ & 0.83 \\
SLE disease duration & $8(8.7)$ & $8.5(11.6)$ & 0.27 \\
Manifestation of SLE & & & \\
$\quad$-Musculoscletal & $75(66.4)$ & $14(63.6)$ & 0.8 \\
$\quad$-Mucocutaneous & $60(52.6)$ & $9(40.9)$ & 0.31 \\
$\quad$-Hematologic & $47(40.9)$ & $10(43.5)$ & 0.49 \\
$\quad$-Serosal & $26(23.2)$ & $4(17.4)$ & 0.54 \\
$\quad$-Neurological & $6(5.3)$ & $1(4.3)$ & 0.85 \\
Laboratory values for kidney biopsy & & & \\
$\quad$-Creatinine level (mg/dL) & $0.7(0.5)$ & $0.9(0.6)$ & 0.17 \\
$\quad$-GFR (ml/min) & $110(67)$ & $77(65)$ & 0.06 \\
$\quad$-24-hour urine protein & & & \\
$\quad \geq 1$ gr/day & $72(71.3)$ & $17(77.3)$ & 0.63 \\
$\quad \geq 3$ gr/day & $36(35.6)$ & $11(50)$ & 0.23 \\
$\quad$-Active urinary sediment & $91(83.5)$ & $6(27.3)$ & $<0.001$ \\
Renal SLEDAl at the biopsy & $12(8)$ & $4(4)$ & $<0.001$ \\
End-stage renal disease & $13(11.2)$ & $2(8.7)$ & 0.72 \\
Renal transplantation & $5(4.3)$ & $1(4.3)$ & 0.99 \\
Exitus & $8(7)$ & $1(4.3)$ & 0.99 \\
\hline
\end{tabular}

" $n$ (\%), if otherwise specified. Med (IQR) for numerical data excluding age; mean $\pm S D$ for age.GFR: Glomerular filtration rate, LN: Lupus nephritis, SLEDAl: Systemic lupus erythematosus disease activity index

Disclosure of Interests: None declared

DOI: 10.1136/annrheumdis-2021-eular.4003

\section{AB0348 ALBUMIN-TO-GLOBULIN RATIO AS A POTENTIAL PREDICTOR OF RENAL REMISSION IN LUPUS NEPHRITIS}

T. Knežević ${ }^{1}$, I. Padjen ${ }^{2}$, S. Bulimbašić ${ }^{3}$, M. Ćorić ${ }^{3}$, V. Ivković ${ }^{1}$, M. Laganović ${ }^{4}$, B. Anić ${ }^{2}{ }^{1}$ University Hospital Centre Zagreb, Division of Nephrology, Hypertension, Dialysis and Transplantation, Department of Internal Medicine, ZAGREB, Croatia; ${ }^{2}$ University Hospital Centre Zagreb and University of Zagreb, School of Medicine, Zagreb, Croatia, Division of Clinical Immunology and Rheumatology, Department of Internal Medicine, ZAGREB, Croatia; ${ }^{3}$ University Hospital Centre Zagreb and University of Zagreb, School of Medicine, Zagreb, Croatia, Department of Pathology and Cytology, University Hospital Centre Zagreb, Croatia, ZAGREB, Croatia; ${ }^{4}$ University Hospital Centre Zagreb and University of Zagreb, School of Medicine, Zagreb, Croatia, Division of Nephrology, Hypertension, Dialysis and Transplantation, National Referral Centre for Glomerular and Tubulointerstitial Diseases, Zagreb, Croatia, ZAGREB, Croatia

Background: Optimal prognostic predictors in lupus nephritis (LN) are still not clearly defined. Studies have recently shown that albumin-to-globulin ratio $(A / G)$ is not only a good predictor of outcomes in malignancy, infection and renal disease, but is also a potential marker of increased immunoglobulin synthesis and LN activity and might be useful as a predictor of the development of LN in SLE patients. Studies on the role of $A / G$ in predicting outcomes in $L N$ are, however, lacking.

Objectives: To assess a potential prognostic value of $A / G$ at renal biopsy in predicting outcomes in LN.

Methods: In this retrospective study we analyzed the demographic, histologic, laboratory and clinical characteristics of patients with biopsy-proven LN diagnosed between 2011 and 2020. All patients met the 1997 revised American
College of Rheumatology classification criteria for SLE. Complete remission was defined as proteinuria $<0.5 \mathrm{~g} /$ day and serum creatinine within $10 \%$ from baseline, and partial remission as more than $50 \%$ reduction in proteinuria to sub-nephrotic levels and serum creatinine within $10 \%$ from baseline.

Results: We have included 55 patients ( $80 \%$ women) with biopsy-proven LN (age at biopsy $38 \pm 13$ years). On renal histology, one patient was class I LN two patients were class II, 7 were class III, 5 were class III+V, 23 were class IV, two were class IV+V, 11 class $\mathrm{VI}$ and 4 had other features. Median eGFR and proteinuria at biopsy were 69 (IQR: 44,100$) \mathrm{mL} / \mathrm{min} / 1.73 \mathrm{~m}^{2}$ and $3.5(2.3,5.6) \mathrm{g}$ day, respectively. Baseline mean $A / G$ was $1.12 \pm 0.29$ and did not differ between LN classes $(p=0.91)$. At one-year follow-up eGFR increased non-significantly (69 vs. $82 \mathrm{~mL} / \mathrm{min} / 1.73 \mathrm{~m}^{2}, \mathrm{p}=0.13$ ) and proteinuria decreased $(3.5$ vs. $0.6 \mathrm{~g} /$ day, $\mathrm{p}<0.001)$. Eighteen patients achieved complete remission (CR), 16 partial remission and 21 did not achieve remission. None progressed to ESRD and two patients died, both from sepsis. A/G at the time of biopsy did not differ between group which achieved CR/PR vs. no remission ( $p>0.05$; Figure 1$)$ and was not a significant predictor of remission in a multivariate regression model (OR 5.9, $95 \% \mathrm{Cl}$ 0.6, 63.2).

Conclusion: While being a potential marker for transition of SLE to $L N, A / G$ was not showed to be a predictor of renal remission in LN. REFERENCES:

[1] Liu XR, et al. Lupus. 2021 Jan 6:961203320981139.

[2] Kwon OC, et al. Semin Arthritis Rheum. 2018 Dec;48(3):462-466ck

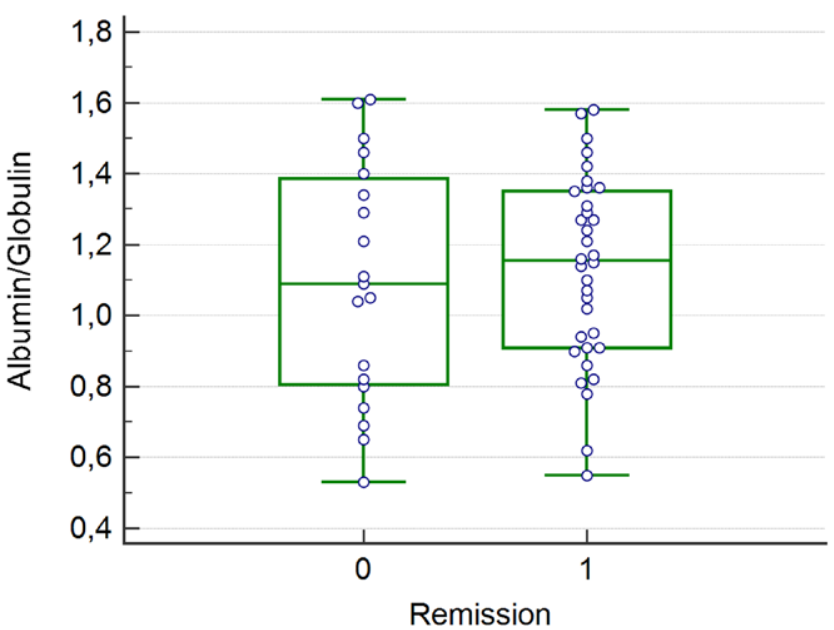

Disclosure of Interests: None declared

DOI: 10.1136/annrheumdis-2021-eular.4111

\section{AB0349 NEUTROPHIL-TO-LYMPHOCYTE RATIO, PLATELET-TO- LYMPHOCYTE RATIO AND MEAN PLATELET VOLUME IN LUPUS NEPHRITIS}

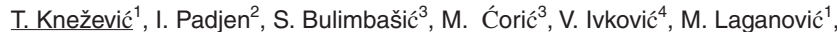
B. Anić ${ }^{2}{ }^{1}$ University Hospital Centre Zagreb and University of Zagreb, School of Medicine, Zagreb, Croatia, Division of Nephrology, Hypertension, Dialysis and Transplantation, Department of Internal Medicine, ZAGREB, Croatia; ${ }^{2}$ University Hospital Centre Zagreb and University of Zagreb, School of Medicine, Zagreb, Croatia, Division of Clinical Immunology and Rheumatology, Department of Internal Medicine, ZAGREB, Croatia; ${ }^{3}$ University Hospital Centre Zagreb and University of Zagreb, School of Medicine, Zagreb, Croatia, Department of Pathology and Cytology, University Hospital Centre Zagreb, Croatia, ZAGREB, Croatia; ${ }^{4}$ University Hospital Centre Zagreb and University of Rijeka, Faculty of Health Studies, Rijeka, Croatia, Division of Nephrology, Hypertension, Dialysis and Transplantation, Department of Internal Medicine, ZAGREB, Croatia

Background: A number of blood count parameters are associated with inflammatory and autoimmune diseases. There is discordant evidence whether neutrophil-to-lymphocyte ratio (NLR), platelet-to-lymphocyte ratio (PLR) and mean platelet volume (MPV) might reflect inflammatory response and have prognostic value in SLE patients. However, the studies examining the potential role of these markers in $\mathrm{LN}$ are missing.

Objectives: To examine NLR, PLR and MPV at the time of biopsy in patients with $L N$ and evaluate their association with prognosis.

Methods: In this retrospective study we analyzed the demographic, histologic laboratory and clinical characteristics of patients with biopsy-proven LN diagnosed between 2011 and 2020. All patients met the 1997 revised American College of Rheumatology classification criteria for SLE. Complete remission was defined as proteinuria $<0.5 \mathrm{~g} /$ day and serum creatinine within $10 \%$ from baseline, 